Check for updates

Cite this: RSC Adv., 2019, 9, 21667

6th June 2019

Accepted 5th July 2019

DOI: 10.1039/c9ra04251b

rsc.li/rsc-advances

\section{The synergistic effect of carbon coating and CNTs compositing on the hard carbon anode for sodium ion batteries $\uparrow$}

\author{
Chang Liu, Jiaqi Chu, Yang Liu, (D) * Yingchun Lyu (D) * and Bingkun Guo*
}

In this work, mesoporous hard carbon materials were synthesized and modified by compositing a carbon coating and carbon nanotubes (CNTs), reducing the surface area and improving the conductivities without changing the microstructures of the anodes, which enhances the coulombic efficiencies and rate performances of sodium-ion batteries (SIBs).

\section{Introduction}

With the increasing concerns over global warming caused by heavy use of fossil fuels, exploring advanced energy storage technologies is becoming urgent which can actively integrate renewable energy into the power grid. ${ }^{1}$ Lithium ion batteries (LIBs), the most commonly used energy storage technology in portable electronics, have been recently expanded to be applied in large-scale energy storage systems due to their high energy density and long cycle life. ${ }^{2}$ However, limited and uneven distribution of lithium resources in Earth's crust will not satisfy such increasing demand for LIBs, which accelerates the development of alternative technologies. ${ }^{3}$ As such, SIBs are deemed to be a promising choice for large-scale energy storage due to the resourceful global distribution and lower price of sodium, ${ }^{4}$ and many Na-based layered oxides or polyanionic compounds can be used as cathodes. ${ }^{5}$

Although sodium has similar chemical properties to lithium, analogous electrode materials do not display equal electrochemical performance because of the large ionic radius of sodium $\left(\mathrm{Li}^{+}: 0.69 \AA \mathrm{Na}^{+}: 0.98 \AA\right) .{ }^{6}$ The difference causes some stumbling blocks in the application of SIBs such as Na-C compounds cannot be formed to store $\mathrm{Na}^{+}$in graphite which is the commercially used anode material in LIBs. ${ }^{7}$ Hard carbon, which has presented good electrochemical performances in alkali metal batteries, ${ }^{8,9}$ is considered to be the most promising anode material for SIBs due to the high reversible capacity, low sodium storage potential, and excellent cycling stability. ${ }^{10}$ Sodium storage mechanism in hard carbon is still not thoroughly understood. The typical galvanostatic curve of hard carbon include two parts, the sloping region $\left(>0.1 \mathrm{~V} v s . \mathrm{Na}^{+} / \mathrm{Na}\right)$ and plateau region $\left(<0.1 \mathrm{~V} v\right.$ s. $\left.\mathrm{Na}^{+} / \mathrm{Na}\right) .{ }^{11}$ Generally, slope region

Materials Genome Institute, Shanghai University, Shanghai, 200444, China. E-mail: liuyang81@shu.edu.cn; guobingkun@shu.edu.cn

$\dagger$ Electronic supplementary information (ESI) available. See DOI: $10.1039 / \mathrm{c} 9 \mathrm{ra} 04251 \mathrm{~b}$ is derived from the sodium adsorption in defected sites, edges, and the surface of nanographitic domains, while the low potential plateau region is mainly corresponding to deposition of sodium in the nanopores of hard carbon..$^{12}$ Therefore, the electrochemical properties of hard carbon are closely related with their microstructures (interlayer spacing, nanopores, surface area and so on). In order to increase their reversible capacity and reduce cost, research efforts of hard carbons have focused on exploring low-cost hard carbon precursors (such as biomass, coal, pitch) and optimizing preparation process. However, there are several issues limited their practical application, such as poor rate performance, which is generally considered to stem from slow sodium diffusion kinetics in the hard carbon particles. ${ }^{13}$

Shorten $\mathrm{Na}^{+}$solid diffusion pathway is an effective approach to improve the rate capability. Inspired by this strategy, various nanostructure materials, such as nanofiber, ${ }^{14}$ nanosheet, ${ }^{15}$ nanoporous carbon materials,$^{16}$ have been developed to enhance their rate performances. Among them, nanoporous materials would be a best choice. ${ }^{17}$ Cao and co-workers synthesized mesoporous carbon by the traditional nano$\mathrm{CaCO}_{3}$ template-assisted method, which displayed high rate capability owing to the nanoporous structure that enable facile diffusion of the liquid electrolyte into the bulk of hard carbon and reduce $\mathrm{Na}^{+}$solid diffusion length. ${ }^{18}$ Zhang et al. reported that $2 \mathrm{D}$ porous carbon sheets, prepared by chemical activation of polypyrrole-functionalized graphene sheets with $\mathrm{KOH}$, revealed a good rate capability $\left(50 \mathrm{~mA} \mathrm{~h} \mathrm{~g}^{-1}\right.$ at a high current density of $\left.20 \mathrm{~A} \mathrm{~g}^{-1}\right) \cdot{ }^{15}$ Introducing other materials, such as carbon nanotubes ${ }^{19}$ or graphenes, ${ }^{20}$ into hard carbons to prepare composites was also considered to be an effective method to improve their rate performances. Pyo et al. found that the incorporation of MWNT changed the micro-structures of hard carbon, which contributed to an improvement in rate capability and reversible capacity. ${ }^{21}$ Dai et al. have proposed to add carbon nanotubes into hard carbon for improving electronic conductivity, which dramatically enhanced their rate 
capability. ${ }^{22}$ Herein, we synthesized mesoporous hard carbon materials modified with carbon coating and incorporation of CNTs additive by soft-templated method, and studied the influence of carbon coating and CNTs additive on electrochemical performances. Basing on the conductivity and cost consideration, MWNT is chosen in this investigation. ${ }^{23}$

\section{Results and discussion}

In this work, CNTs compositing was taken to enhance the conductivity, carbonization temperature was up to $1400{ }^{\circ} \mathrm{C}$ to reduce the defects on carbon surface, carbon coating was taken to regulate the specific surface area, and F127 was used as the soft template to control the pore volume and pore size distribution of mesoporous carbon. Fig. 1 shows the scanning electron microscope (SEM) images of the ME and ME-CNT before and after carbon coating. All the samples present smooth surfaces, suggesting the surface coating layer is uniform. For the hard carbon/CNTs composite samples (Fig. 1c and d), no CNTs can be observed on the surfaces, indicating that the CNTs are well dispersed in the mesoporous carbons. The well dispersed CNTs can provide highly electronic conductive network within the hard carbon, as suggested by the work reported. ${ }^{24}$

To study the structural properties of the samples, XRD and Raman spectroscopy were carried out. As shown in Fig. 2a, two broad peaks at 24 and $43^{\circ}$, typical for disordered carbon, are observed in the XRD patterns. A sharp peak at $26^{\circ}$ can be seen in the XRD pattern of the ME/CNTs composite samples, which is assigned to the CNTs as shown in Fig. 2a. No shift of (002) peak (broad peaks at $24^{\circ}$ ) for ME and ME-CNT samples can be observed, indicating that CNTs addition doesn't alter the interlayer spacing of hard carbon. In Raman spectra (Fig. 2b), two separate characteristic bands of D-band (the defect-induced band) peak at $\sim 1343 \mathrm{~cm}^{-1}$ and G-band (the crystalline graphite band) peak at $\sim 1589 \mathrm{~cm}^{-1}$ are observed, confirming the disordered structure of all the samples. In general, the intensity ratio
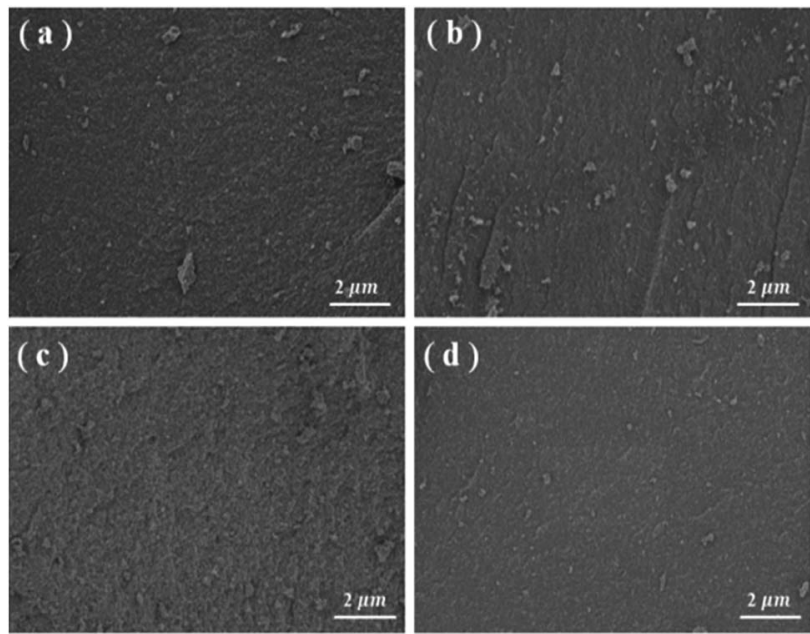

Fig. 1 SEM images of (a) ME; (b) ME-CNT; (c) ME-CC and (d) ME-CNTCC.
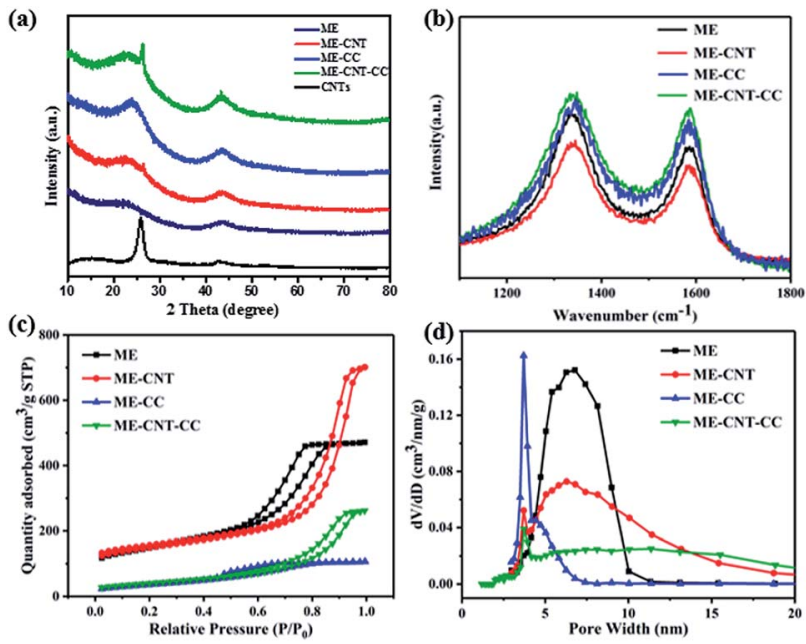

Fig. 2 (a) XRD patterns; (b) Raman spectra; (c) nitrogen adsorptiondesorption isotherms and (d) the pore size distributions of the different samples.

of $\mathrm{D}$ band to $\mathrm{G}$ band $\left(I_{\mathrm{D}} / I_{\mathrm{G}}\right)$ represents the degree of disorder in hard carbon. As shown in Table 1 , the $I_{\mathrm{D}} / I_{\mathrm{G}}$ values of ME and ME-CNT reveals no noticeable change is detected during the treating. XRD and Raman results demonstrate that CNTs addition doesn't distinctly alter microstructures of hard carbon.

The BET surface area and pore size for all samples were measured by $\mathrm{N}_{2}$ adsorption-desorption isotherm. As shown in Fig. 2c and Table 1, both ME and ME-CNT show typical type IV isotherms, indicating highly mesoporous morphology with high surface area of 524 and $531 \mathrm{~m}^{2} \mathrm{~g}^{-1}$, respectively. Obviously the CNTs addition also doesn't change the surface area and pore structure of hard carbon. After surface coating, the mesoporous structure is well-preserved (pore size $\sim 3 \mathrm{~nm}$ ) and the surface area of MPC and MPC-CNT decreases to 134 and $146 \mathrm{~m}^{2}$ $\mathrm{g}^{-1}$, suggesting that the carbon coating layer is uniform. This low surface area may contribute to reduce the formation of solid electrolyte interphase (SEI) and thus improve the initial coulombic efficiency of SIBs.

The electrochemical performances of all samples were tested by coin cells at different measurement conditions. Fig. 3 shows the cyclic voltammetry (CV) curves of ME, ME-CNT, ME-CC, and ME-CNT-CC at a scanning rate of $0.1 \mathrm{mV} \mathrm{s}^{-1}$ in the voltage range of $0-3 \mathrm{~V}$. The irreversible peaks of all the samples at $0.5 \mathrm{~V}$ in the first cathodic process can be assigned to the electrolyte decomposition and SEI formation. It can be clearly seen that the surface coating can effectively decrease the formation of SEI and increase their initial coulombic efficiency by reducing the surface defects and surface area. Moreover, ME-CNT-CC presents the least irreversible reduction current at $\sim 0.5 \mathrm{~V}$, which should be attributed to the synergistic effect of CNTs compositing and carbon coating. Furthermore, a pair of strong redox peaks at around $0.02 / 0.12 \mathrm{~V}$ for all samples can be ascribed to the reversible sodium insertion/extraction in the hard carbons. ${ }^{12}$

The initial galvanostatic discharge-charge profiles of the samples are presented in Fig. $4 \mathrm{a}$ at a current density of $0.1 \mathrm{C}$ (1C 
Table 1 Physical parameters and electrochemical properties for MEs

\begin{tabular}{|c|c|c|c|c|c|}
\hline Material & $d 002[\AA]$ & $I_{\mathrm{D}} / I_{\mathrm{G}}$ ratio & $\mathrm{SA}_{\mathrm{BET}}\left[\mathrm{m}^{2} \mathrm{~g}^{-1}\right]$ & $\mathrm{RC}^{a}\left[\mathrm{~mA} \mathrm{~h} \mathrm{~g}^{-1}\right]$ & $\operatorname{ICE}^{b}[\%]$ \\
\hline ME & 3.95 & 1.20 & 524 & 91.6 & 24.37 \\
\hline ME-CNT & 3.93 & 1.19 & 531 & 113.2 & 25.3 \\
\hline ME-CNT-CC & 3.78 & 1.08 & 146 & 203.6 & 78.39 \\
\hline
\end{tabular}

${ }^{a}$ The reversible capacity (RC). ${ }^{b}$ The initial coulombic efficiency (ICE).
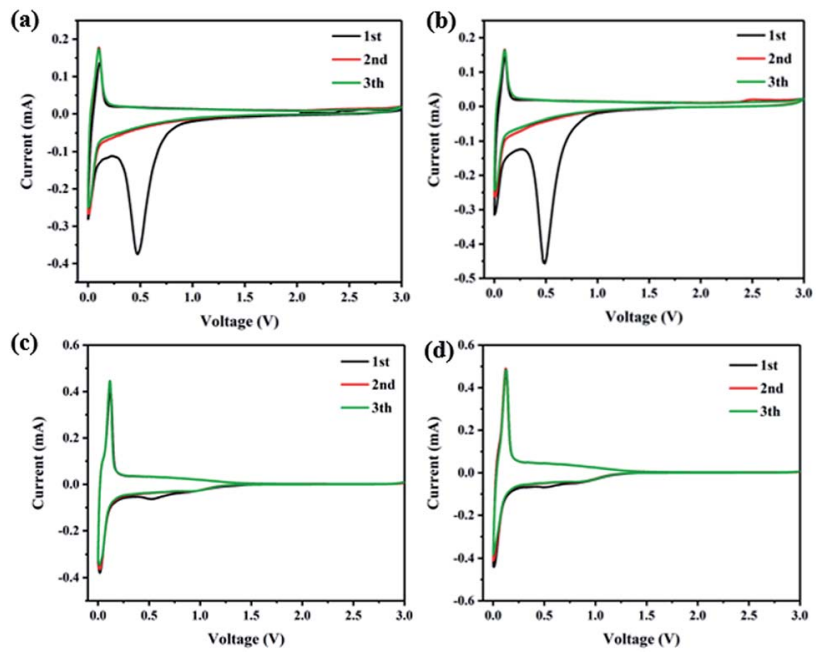

Fig. 3 Cyclic voltammetry (CV) curves of (a) ME, (b) ME-CNT, (c) ME$\mathrm{CC}$ and (d) ME-CNT-CC at $0.1 \mathrm{mV} \mathrm{s}^{-1}$.
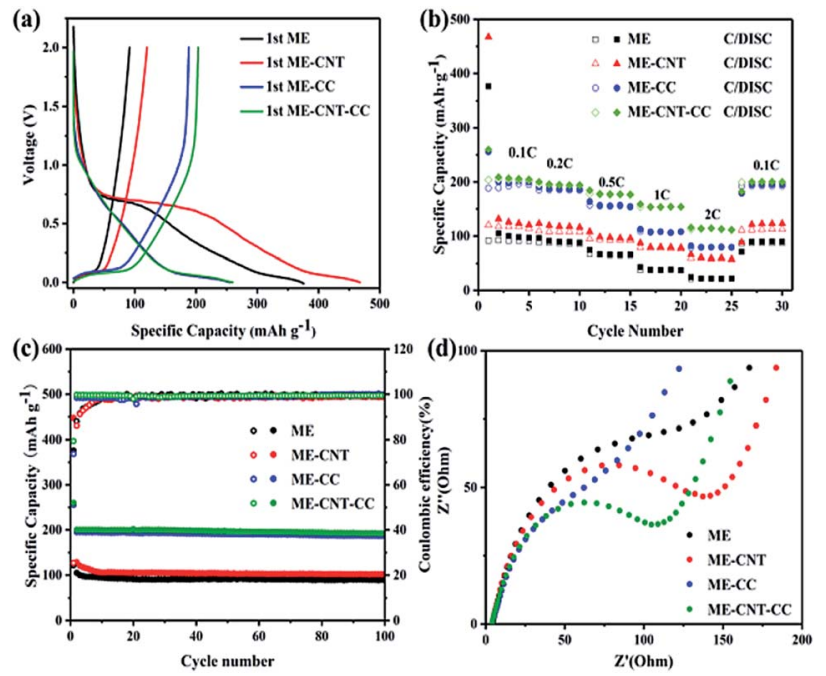

Fig. 4 (a) Galvanostatic initial discharge-charge profiles of the samples at a current rate of $0.1 \mathrm{C}$; (b) rate capabilities of the samples at various current rates from 0.1 to $2 \mathrm{C}$; (c) cyclic performances of the samples at a current rate of $0.1 \mathrm{C}$; (d) Nyquist plots of the samples in 10 cycles.

$=300 \mathrm{~mA} \mathrm{~g}^{-1}$ ). All electrodes exhibit typical two potential regions, the sloping region above $0.1 \mathrm{~V}$ is defined as the $\mathrm{Na}$ adsorption on the surface active sites, such as morphological defects, or the surface. The plateau region around $0.1 \mathrm{~V}$ could be ascribed to the $\mathrm{Na}^{+}$insertion into nanovoids. The electrodes of ME and MPC-CNT deliver reversible specific capacities of 92 and $113 \mathrm{~mA} \mathrm{~h} \mathrm{~g}^{-1}$, and initial coulombic efficiencies of 24.4 and $25.3 \%$, respectively. The large initial capacity loss is mainly attributed to the larger surface area for the formation of SEI. However, there is a significant increase in the specific capacity and initial coulombic efficiency of the material after carbon coating. The electrodes of ME-CC and ME-CNT-CC deliver reversible specific capacities of 188 and $204 \mathrm{~mA} \mathrm{~h} \mathrm{~g}^{-1}$, and initial coulombic efficiencies of 73.7 and $78.4 \%$, respectively. The results indicate that carbon coating is an effective method to reduce the irreversible sodium loss, thereby achieving the purpose of improving the initial coulombic efficiency and specific capacity. Furthermore, the hard carbon samples with CNTs displays longer low potential plateau and higher reversible capacity than the samples without CNTs, suggesting that the CNTs additive can improve the kinetics of hard carbon.

Fig. $4 \mathrm{~b}$ exhibits rate performances of the samples at various current rates from 0.1 to $2 \mathrm{C}$. All the surfaces coated samples exhibit good rate performances. For example, the specific capacities of ME-CNT-CC are $154 \mathrm{~mA} \mathrm{~h} \mathrm{~g}{ }^{-1}$ at 1C and $116 \mathrm{~mA} \mathrm{~h} \mathrm{~g}{ }^{-1}$ at $2 \mathrm{C}$, respectively. The improvements in rate capabilities of the samples after carbon coating could be ascribed to the limited SEI formation on their surfaces. Furthermore, the rate performance of mesoporous hard carbon is also improved by addition of CNTs. The reversible capacities of the carbon/CNTs composite are retained at 80 and $60 \mathrm{~mA} \mathrm{~h} \mathrm{~g}{ }^{-1}$ at current density of 1C and 2C, respectively. As mentioned above, no microstructure and pore structure change can be observed from the results of XRD, Raman and $\mathrm{N}_{2}$ adsorption-desorption isotherm, demonstrating that the high reversible capacity and high rate capability of the samples with CNTs could be attributed to enhanced electronic conductivity in hard carbon samples after CNTs addition. Besides good rate capabilities, ME-CC and ME-CNT-CC samples reveal excellent cycling stability. As shown in Fig. 4c, the capacity retention of both samples is as high as $97 \%$ in 100 cycles. It can be seen that the better electrochemical performance of the ME-CNT-CC results from the combination of fast ion transport of mesoporous structure and high electron transport of CNTs within the hard carbon. Moreover, ME-CC also presents a good cyclic performance in Fig. 4c, which should be related to relative low rate at $0.1 \mathrm{C}$. When the discharge rate is $0.12 \mathrm{C}\left(0.1 \mathrm{mV} \mathrm{s}^{-1}\right.$ in Fig. 3c) and 0.2-2C (Fig. 4b), ME-CNT-CC all presents the best discharge capacities. The electrochemical impedance 
spectroscopy (EIS) of all samples in 10 cycles are shown in Fig. $4 \mathrm{~d}$. The similar contact impedance mean the coating did not affect the bulk conductive of the samples, and the clearly smallest charge transfer impedance of the MPC-CNT-CC further demonstrates the synergistic effect of electronic conductive additive and surface coating.

\section{Conclusions}

In summary, the effects of carbon coating and CNTs compositing on mesoporous hard carbon were investigated. The carbon coating reduced the surface area of hard carbon from 524 and $531 \mathrm{~m}^{2} \mathrm{~g}^{-1}$ to 134 and $146 \mathrm{~m}^{2} \mathrm{~g}^{-1}$, which improved the coulombic efficiency of the electrode from 25.3 to $78.4 \%$, and the well dispersed CNTs would provide good conductive network in the hard carbons without altering their microstructure. With the synergistic effect of surface coating and CNTs compositing, the sample presents a discharge capacity of $203 \mathrm{~mA} \mathrm{~h} \mathrm{~g}^{-1}$ at $0.1 \mathrm{C}$, retains $97 \%$ in 100 cycles, and improves the rate performance from 23 to $116 \mathrm{~mA} \mathrm{~h} \mathrm{~g}^{-1}$ at $2 \mathrm{C}$, suggesting a highly efficient and easily scale-up approach to elevate hard carbons as the anode for SIBs. The knowledge obtained in this work is useful to guide the design and preparation of the hard carbon anodes for $\mathrm{Na}$ ion batteries.

\section{Conflicts of interest}

There are no conflicts to declare.

\section{Acknowledgements}

This work was supported by the National Natural Science Foundation of China (51602190) and the 111 Project (D16002).

\section{References}

1 J. B. Goodenough, Energy Storage Materials, 2015, 1, 158-161.

2 J. B. Goodenough and K.-S. Park, J. Am. Chem. Soc., 2013, 135, 1167-1176.

3 Y. Li, Y. Lu, C. Zhao, Y.-S. Hu, M.-M. Titirici, H. Li, X. Huang and L. Chen, Energy Storage Materials, 2017, 7, 130-151.

4 D. Kundu, E. Talaie, V. Duffort and L. F. Nazar, Angew. Chem., Int. Ed., 2015, 54, 3431-3448.

5 Y. Huang, Y. Zheng, X. Li, F. Adams, W. Luo, Y. Huang and L. Hu, ACS Energy Lett., 2018, 3, 1604-1612.
6 V. Palomares, P. Serras, I. Villaluenga, K. B. Hueso, J. Carretero-González and T. Rojo, Energy Environ. Sci., 2012, 5, 5884-5901.

7 Y. Liu, B. V. Merinov and W. A. Goddard, Proc. Natl. Acad. Sci. U. S. A., 2016, 113, 3735-3739.

8 Z. Wu, L. Wang, J. Huang, J. Zou, S. Chen, H. Cheng, C. Jiang, P. Gao and X. Niu, Electrochim. Acta, 2019, 306, 446-453.

9 L. Wang, J. Yang, J. Li, T. Chen, S. Chen, Z. Wu, J. Qiu, B. Wang, P. Gao, X. Niu and H. Li, J. Power Sources, 2019, 409, 24-30.

10 D. A. Stevens and J. R. Dahn, J. Electrochem. Soc., 2000, 147, 1271.

11 Y. Li, Y. S. Hu, H. Li, L. Chen and X. Huang, J. Mater. Chem. A, 2015, 4, 96-104.

12 C. Bommier, T. Wesley Surta, M. Dolgos and X. Ji, Nano Lett., 2015, 15, 5888-5892.

13 D. Li, H. Chen, G. Liu, M. Wei, L. Ding, S. Wang and H. Wang, Carbon N Y, 2015, 94, 888-894.

14 W. Luo, J. Schardt, C. Bommier, B. Wang, J. Razink, J. Simonsen and X. Ji, J. Mater. Chem. A, 2013, 1, 1066210666.

15 H. Wang, W. Zhong, M. Fan-lu, M. De-long, H. Xiao-lei, L. Wang and X. Zhang, ChemSusChem, 2013, 6, 56-60.

16 S. J. R. Prabakar, J. Jeong and M. Pyo, Electrochim. Acta, 2015, 161, 23-31.

17 P. Lu, Y. Sun, H. Xiang, X. Liang and Y. Yu, Adv. Energy Mater., 2018, 8, 1702434.

18 B. Cao, H. Liu, B. Xu, Y. Lei, X. Chen and H. Song, J. Mater. Chem. A, 2016, 4, 6472-6478.

19 X. F. Luo, C. H. Yang, Y. Y. Peng, N. W. Pu, M. Der Ger, C. Te Hsieh and J. K. Chang, J. Mater. Chem. A, 2015, 3, 1032010326.

20 Y. Yan, Y.-X. Yin, Y.-G. Guo and L.-J. Wan, Adv. Energy Mater., 2014, 4, 1301584.

21 R. Suresh Babu and M. Pyo, J. Electrochem. Soc., 2014, 161, A1045-A1050.

22 B. Guo, X. Wang, P. F. Fulvio, M. Chi, S. M. Mahurin, X.-G. Sun and S. Dai, Adv. Mater., 2011, 23, 4661-4666.

23 R. C. Y. King, F. Roussel, J.-F. Brun and C. Gors, Synth. Met., 2012, 162, 1348-1356.

24 G. Hasegawa, K. Kanamori, N. Kannari, J. Ozaki, K. Nakanishi and T. Abe, J. Power Sources, 2016, 318, 41-48. 